\title{
Comorbid conditions and the transition among states of hip osteoarthritis and symptoms in a community-based study: a multi-state time-to-event model approach
}

Carolina Alvarez', Rebecca J. Cleveland ${ }^{1}$, Todd A. Schwartz ${ }^{1,2}$, Jordan B. Renner ${ }^{1,3}$, Louise B. Murphy ${ }^{4}$, Joanne M. Jordan ${ }^{1,5,6}$, Leigh F. Callahan ${ }^{1,5,6}$, Yvonne M. Golightly ${ }^{1,6,7,8}$ and Amanda E. Nelson ${ }^{1,5^{*}}$ (D)

\begin{abstract}
Background: We examined the association of three common chronic conditions (obesity, diabetes mellitus [DM], and cardiovascular disease [CVD]) with transitions among states of hip osteoarthritis (HOA).

Methods: This longitudinal analysis used data from the Johnston County Osteoarthritis Project (JoCo OA, $n=3857$ ), a community-based study in North Carolina, USA, with 18.4 1.5 years of follow-up. Transitions across the following states were modeled: development of radiographic $\mathrm{HOA}$ ( $\mathrm{rHOA}$; Kellgren-Lawrence grade $[\mathrm{KLG}$ of $<2$ ); development of hip symptoms (self-reported hip pain, aching, or stiffness on most days) or symptomatic HOA (sxHOA; rHOA and symptoms in the same hip), and resolution of symptoms. Obesity (body mass index $\geq 30 \mathrm{~kg} / \mathrm{m}^{2}$ ) and self-reported DM and CVD were the time-dependent comorbid conditions of interest. Markov multi-state models were used to estimate adjusted hazard ratios and 95\% confidence intervals to describe the associations between the conditions and HOA states.

Results: The sample included 33\% African Americans, 39\% men, with a mean (SD) age of 62.2 (9.8) years; the frequencies of the comorbidities increased substantially over time. When considered individually, obesity was associated with incident hip symptoms, while CVD and DM were associated with reduced symptom resolution. For those with $>1$ comorbidity, the likelihood of incident sxHOA increased, while that of symptom resolution significantly decreased. When stratified by sex, the association between obesity and incident symptoms was only seen in women; among men with DM versus men without, there was a significant $(\sim 75 \%)$ reduction in symptom resolution in those with $\mathrm{rHOA}$. When stratified by race, African Americans with $\mathrm{DM}$, versus those without, were much more likely to develop sxHOA.

Conclusions: Comorbid chronic conditions are common in individuals with $\mathrm{OA}$, and these conditions have a significant impact on the persistence and progression of HOA. OA management decisions, both pharmacologic and non-pharmacologic, should include considerations of the inter-relationships between $\mathrm{OA}$ and common comorbidities such as DM and CVD.
\end{abstract}

Keywords: Hip osteoarthritis, Comorbidities, Race and gender differences

\footnotetext{
* Correspondence: aenelson@med.unc.edu

${ }^{1}$ Thurston Arthritis Research Center, University of North Carolina at Chapel Hill, 3300 Doc J. Thurston Building, Campus Box \#7280, Chapel Hill, NC 27599-7280, USA

${ }^{5}$ School of Medicine, University of North Carolina at Chapel Hill, Chapel Hill, USA

Full list of author information is available at the end of the article
}

(c) The Author(s). 2020 Open Access This article is distributed under the terms of the Creative Commons Attribution 4.0 International License (http://creativecommons.org/licenses/by/4.0/), which permits unrestricted use, distribution, and reproduction in any medium, provided you give appropriate credit to the original author(s) and the source, provide a link to the Creative Commons license, and indicate if changes were made. The Creative Commons Public Domain Dedication waiver (http://creativecommons.org/publicdomain/zero/1.0/) applies to the data made available in this article, unless otherwise stated. 


\section{Background}

Osteoarthritis (OA) in general has been associated with a substantially higher risk of cardiovascular disease (CVD) [1] and of premature mortality [2], although some of this increased risk is likely explained by walking disability [3]. Conditions such as CVD and diabetes mellitus (DM) are commonly comorbid with OA and have been associated with poorer outcomes, for example following joint replacement [4]. Hip OA (HOA) is a common chronic condition, which will affect a quarter of the population by age 85 [5]. The evidence that OA is associated with CVD, DM, or features of the metabolic syndrome (e.g., hyperglycemia, insulin resistance, obesity, and dyslipidemia) is mixed and is overall stronger for knee OA compared with HOA [6]. The evidence of a specific association between HOA and CVD remains undecided [7], with few studies focused on DM.

Similarly, while obesity is a clear and well-known risk factor for knee OA, its relationship to HOA is less established. The majority of cohort studies to date have found strong associations between obesity and knee OA but no or modest ones for HOA [8]. A 2011 systematic review of 14 studies reported a significant but modest positive association between body mass index (BMI) and HOA, where HOA risk rose by $10 \%$ with each increasing unit $\left(\mathrm{kg} / \mathrm{m}^{2}\right)$ of BMI (risk ratio of 1.10 [95\% confidence interval 1.07-1.16]) [9]. However, most previous studies were cross-sectional, limiting the ability to determine cause and effect. The Johnston County OA Project has extensive longitudinal data on $\mathrm{HOA}$, including symptoms and radiographs and the presence and development of obesity and comorbid conditions, including DM and CVD. Using this unique dataset, we aimed to determine the associations between prevalent or incident obesity, DM, and CVD and the transitions among key states of HOA (e.g., development or resolution of symptoms, or development of radiographic damage).

\section{Patients and methods}

\section{Study participants}

The study sample, drawn from a community-based, prospective observational cohort of civilian, noninstitutionalized African American and white men and women in Johnston County, North Carolina, USA, consisted of an original cohort (baseline data collection 1991-1997) and enrichment cohort (baseline data collection 2003-2004, enrolled to replace losses from the original cohort over time), as previously described [10]; this study has been approved by the University of North Carolina (IRB 920583). All participants were at least 45 years of age at enrollment, although women under 50 years of age did not undergo pelvis radiography per protocol; pelvis radiography for women was added at the visit where they were 50 years of age or older (which was considered their baseline visit). Follow-up data were collected during
1999-2003 for the original cohort, and 2006-2011 and 2013-2015 for both the original and enrichment cohorts (Fig. 1). Vital status for all participants was assessed through the National Death Index up to December 31, 2015. From the initial study sample from both cohorts of 3919 participants with hip X-rays and mortality data, less than $2 \%(n=62)$ were missing at least one baseline covariate and were excluded. A complete case analysis was conducted on the remaining 3857 individuals in the analytic sample. Of the additional participants who were lost to follow-up, about 2/5 were due to lack of interest, with the remainder evenly split among the following: moving out of the study area, being physically/mentally unable to participate, or inability to contact. These participants were generally younger, less educated, and more often from the enrichment cohort. A sensitivity analysis limited to those with at least two follow-up time points was performed to assess the impact of loss to follow-up.

\section{Outcomes: rHOA and symptom assessment}

Participants were classified as having the following outcomes of interest, if present in at least one hip: (1) radiographic $\mathrm{HOA}$ (rHOA) as Kellgren-Lawrence grade $\geq 2$; (2) hip symptoms defined by self-reported hip pain, aching, or stiffness on most days; (3) symptomatic hip osteoarthritis (sxHOA) defined by both rHOA and symptoms in the same hip. In cases where the hips were disparate within a person, rHOA status was considered first (e.g., if a participant had one hip with symptoms but no rHOA and one hip with asymptomatic rHOA, that person was classified as asymptomatic rHOA). The states of transition modeled were (1) neither rHOA nor hip symptoms (state A); (2) asymptomatic rHOA (rHOA without symptoms, state B); (3) hip symptoms only (symptoms without rHOA, state C); (4) sxHOA (state D); (5) death (state E) as an absorbing state, i.e., a state that cannot be left once entered (Fig. 2). Hip replacements were infrequent in this cohort (at baseline, 15 participants with at least one THR; subsequent incident THRs in 17, 37, and 21 participants by the first, second, and third follow-ups, respectively, for a total of 90) and were included in the analysis as having either rHOA (if no symptoms present) or sxHOA (if symptoms were present).

\section{Main effects: comorbid conditions}

The three comorbidities were defined separately at each study visit. Obesity was defined as a measured BMI of at least $30.0 \mathrm{~kg} / \mathrm{m}^{2}$. For self-reported conditions, participants were read the following statement: "Please tell me which of the following conditions or illnesses a doctor, nurse, or health professional has told you that you have now or have ever had." Self-reported DM status was elicited through a yes/no answer to "diabetes or high blood sugar." Across data collection cycles, CVD status was 


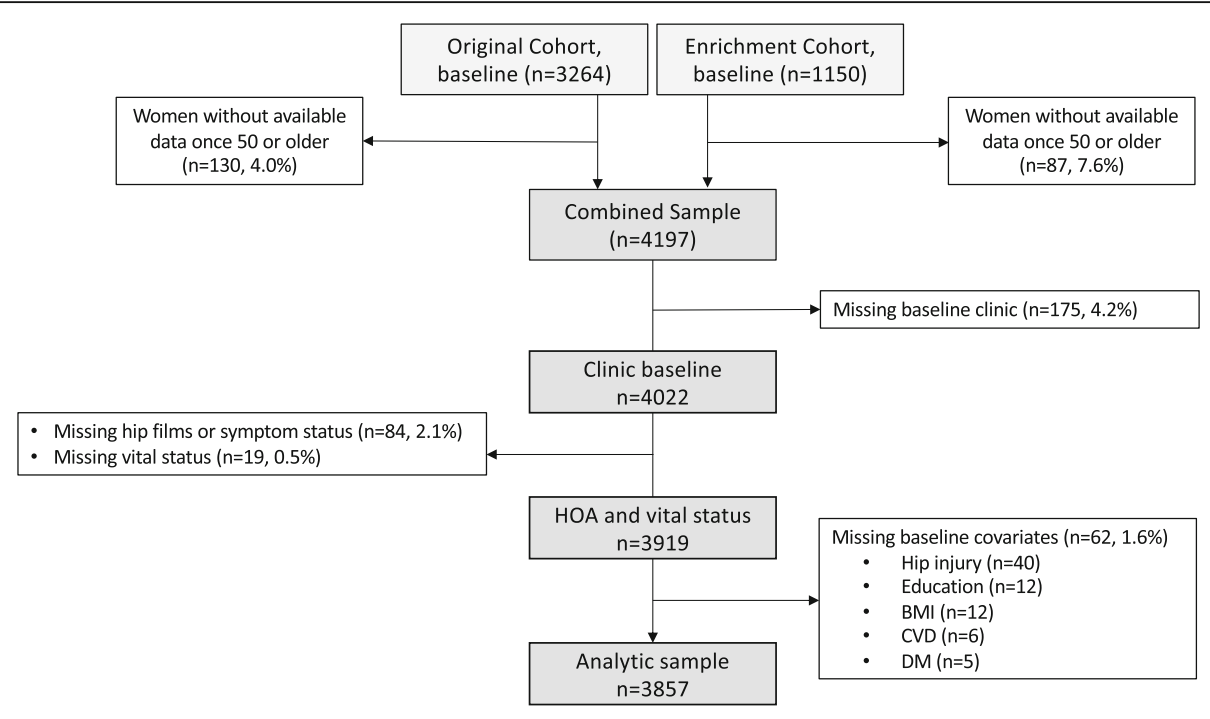

Fig. 1 Flowchart of participant inclusion/exclusion at baseline

assessed with increasing inclusiveness and specificity (baseline: heart attack, other heart problems, cerebrovascular accident; first follow-up: added angina, congestive heart failure; second follow-up: added peripheral vascular disease). All comorbidities were assessed as timedependent, meaning that their presence could change across time for a given participant; for obesity, both development and resolution were possible, but DM and CVD could only develop and not resolve.

\section{Statistical analysis}

To meet this study's objectives, we chose an advanced method to allow modeling of several transitions of interest, which incorporated varying or unequally spaced times of transition, and included covariates which changed over time. The models include transitions across multiple events including condition worsening and improvement. A time-to-event analysis was performed using data from baseline and up to three follow-up time points. Markov multi-state models (MSM) for intervalcensored outcomes (i.e., outcomes occurring during the interval between assessment time points) were conducted using $\mathrm{R}$ software and the MSM package. [11] MSM are based on the theory of stochastic processes, which describe a collection of random variables representing the evolution of a process over time. They assess

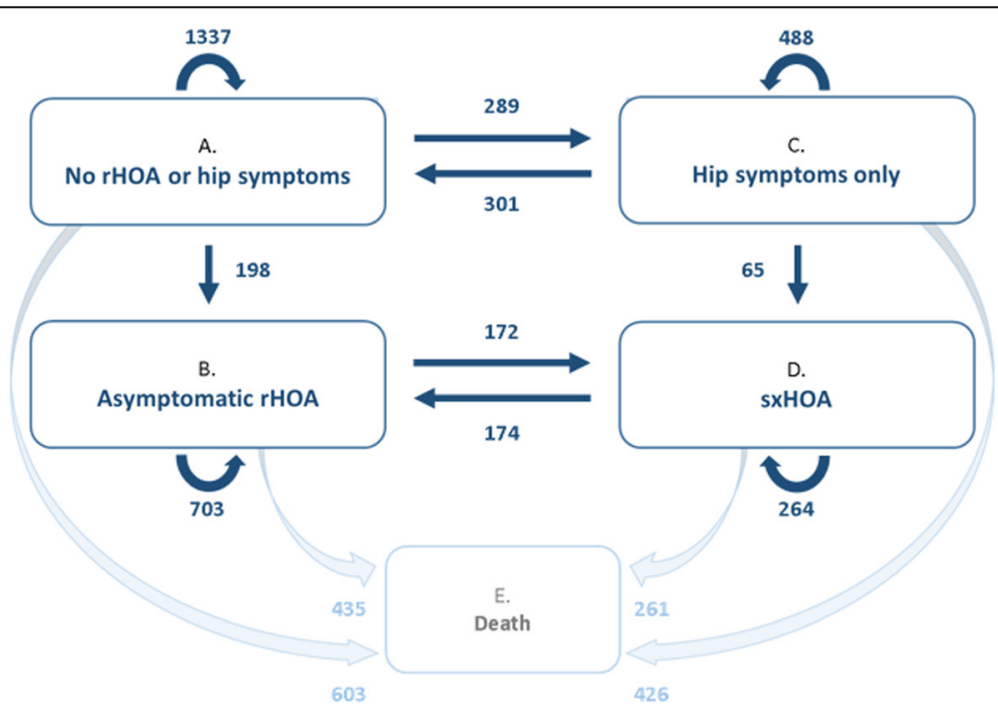

Fig. 2 Five-state progressive model for hip status. The number of times each pair of states was observed at successive follow-up times is indicated next to its respective transition arrow. Numbers indicate number of transition instances, not individuals, over the full follow-up period. Diagonal states, while possible, were infrequent and were excluded for parsimony 
how an individual (rather than a hip) transitions between states in continuous time under the Markov assumption, where future transition depends only on the current state. We used time-inhomogeneous, piecewise exponential models to model and change intensities for all participants at age 65 ; in other words, the exponential parametric model was assumed constant at two separate intervals (while $<65$ or while 65 years or older), recognizing that the standard assumption of constant risk for these transitions does not capture the known effect of increased age. Therefore, in addition to adjusting the model for age, the estimates were allowed to change based on a threshold at age 65; given the clinical and sample-based (near the median) plausibility of this threshold, no other age thresholds were used.

Adjusted hazard ratios (aHRs) and corresponding 95\% confidence intervals ( $95 \% \mathrm{CI})$ were estimated to determine independent associations between each comorbidity and each hip state transition, individually and in combination, in a five-state progressive model. Pairwise combination models were used to determine effects when each pair of comorbidities occurred concurrently, compared to the absence of those two comorbidities. A separate combination model was used to determine transition effects when all three comorbidities were present, compared to the absence of all three comorbidities. The transitions modeled using MSM were development of rHOA (either $\mathrm{A}$ to $\mathrm{B}$ or $\mathrm{C}$ to $\mathrm{D}$ shown in Fig. 2), development of symptoms (A to $C$ or $B$ to $D$ ), or resolution of symptoms ( $\mathrm{C}$ to $\mathrm{A}$ or $\mathrm{D}$ to $\mathrm{B})$. While diagonal transitions are also possible, these were infrequent (35 instances of transition from states A to D, 64 transitions from states $\mathrm{C}$ to $\mathrm{B}$ ), indicating a transitional state with the final event being rHOA, and thus were dropped from the model for simplicity. Additionally, transitions to death (the absorbing state) were modeled but results of main effects on these transitions are not shown as this was not the aim of our study. Qualitative model assessment was conducted by visual consideration of observed and expected prevalence plots for each state. We adjusted for covariates assumed to be either static or changing at observed times (if time-dependent). All models were person-based and were adjusted for relevant baseline demographics (age, sex, race, and education $[<12$ years $]$ ), self-reported, time-dependent history of hip injury or fracture, cohort (original or enrichment), and mean-centered birth year. Stratification by race and sex was performed for each of the individual comorbidities in an exploratory manner given sample size limitations (which did not permit stratified analyses of combinations of comorbidities).

\section{Results}

Descriptive results

The three follow-up visits took place approximately every 6 years. At baseline, the sample included 33\%
African Americans, 39\% men, with a mean (SD) age of 62.2 (9.8) years, and $37 \%$ with less than 12 years of education (Table 1). At that time, $45 \%$ had no rHOA or hip symptoms, $25 \%$ had hip symptoms only, $19 \%$ had asymptomatic rHOA, and $11 \%$ had sxHOA. By the end of the follow-up period (i.e., third follow-up), asymptomatic rHOA had increased to $34 \%$ and sxHOA had increased to $15 \%$. In other words, of the 1743 participants starting without $\mathrm{rHOA}$ or symptoms, $26 \%$ (452) went on to transition to states of hip OA, symptoms, or both. The rest $(n=1291)$ did not transition by the third follow-up visit, having died $(n=748)$ or being censored $(n=543)$. Only $6 \%$ reported a hip injury or fracture at baseline, which doubled over the follow-up period. The majority of the sample reported no symptoms in their hips at all time points, although all categories from mild to severe were represented (Table 1); women were more likely to report symptoms than men, with no differences by race (data not shown). Forty percent of the baseline sample met criteria for obesity, increasing to nearly $50 \%$ by the last follow-up. Similarly, the percentage of both DM and CVD approximately doubled over time (from 14 to $28 \%$ for DM and 22 to $48 \%$ for CVD), although the cumulative incidence over each time point was relatively stable $(9-10 \%$ for DM and $11-13 \%$ for CVD, Table 1).

\section{Associations among HOA state transitions and individual comorbidities}

First, we considered the overall effect of each comorbid condition (i.e., obesity, DM, and CVD) at each visit on the transitions across states of $\mathrm{HOA}$ at subsequent visits (Table 2). Compared with individuals without obesity, those with obesity had a significant $33 \%$ higher hazard of developing symptoms (states A to C) over the full follow-up period. The association for obesity and development of incident sxHOA was also positive although not statistically significant (aHR 1.46, 95\% CI $[0.91,2.36])$. Compared to those without CVD, those with or who developed CVD were more likely to develop asymptomatic rHOA (A to B) or to develop symptoms (A to C), although neither association was statistically significant. However, among those with symptoms only, those with CVD were significantly less likely to have symptom resolution than those without CVD (C to A); a similar trend was seen for symptom resolution in those with $\mathrm{rHOA}$ although not statistically significant. Having or developing DM was not significantly associated with any of the overall transitions, but similar to CVD, symptoms were less likely to resolve among those with DM compared to those without (states C to A: aHR 0.74, 95\% CI [0.51, 1.08] and states D to B: aHR 0.64, 95\% CI $[0.38,1.08])$. 
Table 1 Descriptive characteristics of JoCo OA participants with complete data $(n=3857)$

\begin{tabular}{|c|c|c|c|c|c|c|c|c|}
\hline \multirow[b]{3}{*}{ Characteristicst } & \multicolumn{8}{|c|}{ Study visit (mean \pm SD years from baseline) } \\
\hline & \multicolumn{2}{|c|}{$\begin{array}{l}\text { Baseline } \\
n=3857\end{array}$} & \multicolumn{2}{|c|}{$\begin{array}{l}1 \text { 1st follow-up } \\
n=2300 \\
(6.0 \pm 1.2 \text { years })\end{array}$} & \multicolumn{2}{|c|}{$\begin{array}{l}\text { 2nd follow-up } \\
n=1336 \\
\text { (12.2 } \pm 1.5 \text { years) }\end{array}$} & \multicolumn{2}{|c|}{$\begin{array}{l}\text { 3rd follow-up } \\
n=454 \\
(18.4 \pm 1.5 \text { years })\end{array}$} \\
\hline & $n$ & $\%$ & $n$ & $\%$ & $n$ & $\%$ & $n$ & $\%$ \\
\hline Age (years, mean \pm SD)) & 62.2 & \pm 9.8 & 67.0 & \pm 9.2 & 71.1 & \pm 7.8 & 74.9 & \pm 6.7 \\
\hline Original cohort (vs enrichment) & 2732 & 70.8 & 1617 & 70.3 & 960 & 71.9 & 454 & 100 \\
\hline Men & 1495 & 38.8 & 808 & 35.1 & 461 & 34.5 & 189 & 41.6 \\
\hline African American & 1269 & 32.9 & 710 & 30.9 & 403 & 30.2 & 114 & 25.1 \\
\hline$<12$ years education & 1412 & 36.6 & 701 & 30.5 & 298 & 22.3 & 68 & 15.0 \\
\hline Hip injury & 245 & 6.4 & 200 & 8.7 & 125 & 9.4 & 52 & 11.5 \\
\hline Obesity (BMI $\geq 30 \mathrm{~kg} / \mathrm{m}^{2}$ ) & 1543 & 40.0 & 1061 & 46.1 & 663 & 49.6 & 222 & 48.9 \\
\hline Prevalent DM & 532 & 13.8 & 457 & 19.9 & 340 & 25.4 & 127 & 28.0 \\
\hline Incident DM & & & 196 & 8.5 & 114 & 8.5 & 43 & 9.5 \\
\hline Prevalent CVD & 855 & 22.2 & 734 & 31.9 & 557 & 41.7 & 217 & 47.8 \\
\hline Incident CVD & & & 302 & 13.1 & 159 & 11.9 & 50 & 11.0 \\
\hline \multicolumn{9}{|l|}{ Hip symptoms (max) } \\
\hline None & 2403 & 62.3 & 1512 & 65.7 & 928 & 69.5 & 300 & 66.1 \\
\hline Mild & 405 & 10.5 & 251 & 10.9 & 137 & 10.3 & 46 & 10.1 \\
\hline Moderate & 645 & 16.7 & 343 & 14.9 & 181 & 13.5 & 44 & 9.7 \\
\hline Severe & 375 & 9.7 & 194 & 8.4 & 90 & 6.7 & 64 & 14.1 \\
\hline \multicolumn{9}{|l|}{ State definitions } \\
\hline No rHOA or symptoms & 1743 & 45.2 & 947 & 41.2 & 531 & 39.7 & 160 & 35.2 \\
\hline Asymptomatic rHOA & 736 & 19.1 & 564 & 24.5 & 421 & 31.5 & 154 & 33.9 \\
\hline Hip symptoms only & 967 & 25.1 & 505 & 22.0 & 201 & 15.0 & 71 & 15.6 \\
\hline SXHOA & 411 & 10.7 & 284 & 12.3 & 183 & 13.7 & 69 & 15.2 \\
\hline
\end{tabular}

$B M I$ body mass index in $\mathrm{kg} / \mathrm{m}^{2}$, measured and calculated from height and weight, $D M$ diabetes mellitus, and CVD cardiovascular disease, are self-reported (see "Main effects: comorbid conditions" for additional detail. OA osteoarthritis, rHOA radiographic hip OA, sxHOA symptomatic hip OA t $n(\%)$ unless otherwise noted

${ }^{\$} n=29$ participants are missing at baseline (this was a separate question than the ANY symptoms question used for the state definitions). The maximum severity for the person, considering both hips, is shown

\section{Associations among HOA state transitions and multiple comorbidities}

When the comorbidities were instead considered jointly (Table 2), the combination of obesity and CVD (compared with the absence of both conditions) nearly doubled the rate of symptom worsening (states $\mathrm{A}$ to $\mathrm{C}$ ), while also reducing the rate of symptom resolution by half among those without rHOA (states C to A). Symptom resolution among those with both conditions and rHOA was also reduced but was not statistically significant (states D to B, aHR 0.62, 95\% CI $[0.35,1.10]$ ). The combination of obesity and DM, or of CVD and DM, compared with the absence of both conditions, resulted in more than twice the risk of developing $\mathrm{rHOA}$ in those with symptoms (incident sxHOA, states $\mathrm{C}$ to $\mathrm{D}$ ) and was statistically significant. These combinations (obesity and DM, or CVD and DM) also resulted in significantly lower risk of symptom resolution among those with or without $\mathrm{rHOA}$ (states $\mathrm{D}$ to $\mathrm{B}$ and $\mathrm{C}$ to $\mathrm{A}$, respectively,
Table 2). Additionally, when considering all three comorbidities versus none in a combined model, similar patterns were seen, in that individuals with obesity, DM, and CVD compared to those with none of these comorbidities were substantially less likely to experience symptomatic resolution regardless of rHOA status (states $\mathrm{C}$ to A, aHR $0.39,95 \%$ CI $[0.18,0.83]$; states D to B, aHR $0.22,95 \%$ CI $[0.08,0.60])$, and had nearly four times the likelihood of developing incident sxHOA (state C to D, aHR 3.71, 95\% CI [1.44, 9.58]; data not shown).

\section{Exploratory stratified analyses}

When stratified by sex, the overall pattern was similar, with a few notable differences (Table 3). Although in the same direction, the association between obesity and development of symptoms (states A to C) was significant in women (aHR 1.44, 95\% CI [1.02, 2.02]) but not in men (aHR 1.15, 95\% CI $[0.74,1.79]$ ), as was the statistically non-significant but suggestive association between 
Table 2 Adjusted hazard ratios (aHR) and 95\% confidence intervals (CI) for comorbid conditions, individually and in combination, on modeled transition states, over the full follow-up period

\begin{tabular}{|c|c|c|c|c|c|c|}
\hline \multirow[t]{4}{*}{ Type of transition } & \multicolumn{3}{|c|}{ Individual comorbid conditions } & \multicolumn{3}{|c|}{ Combinations of comorbid conditions } \\
\hline & $\begin{array}{l}\text { Obesity (vs } \\
\text { no obesity) }\end{array}$ & $\begin{array}{l}\text { DM (vs } \\
\text { no DM) }\end{array}$ & $\begin{array}{l}\text { CVD (vs } \\
\text { no CVD) }\end{array}$ & $\begin{array}{l}\text { Obesity and CVD (vs: no } \\
\text { Obesity and no CVD) }\end{array}$ & $\begin{array}{l}\text { Obesity and DM (vs: no } \\
\text { Obesity and no DM) }{ }^{3}\end{array}$ & $\begin{array}{l}\text { CVD and DM (vs: no } \\
\text { CVD and no DM) }{ }^{4}\end{array}$ \\
\hline & \multicolumn{3}{|c|}{$n($ vs $n)$ for transitions } & \multicolumn{3}{|l|}{$n($ vs $n)$ for transitions } \\
\hline & \multicolumn{3}{|l|}{ aHR $(95 \% \mathrm{Cl})$} & \multicolumn{3}{|l|}{ aHR $(95 \% \mathrm{Cl})$} \\
\hline \multicolumn{7}{|l|}{ Development of rHOA } \\
\hline \multirow{2}{*}{$\begin{array}{l}\text { No rHOA/symptoms } \\
\text { (A) to rHOA (B) }\end{array}$} & 82 (vs 116) & 39 (vs 159) & 69 (vs 129) & 16 (vs 75) & 12 (vs 103) & 6 (vs 103) \\
\hline & $0.88(0.65,1.18)$ & $1.06(0.68,1.63)$ & $1.34(0.97,1.85)$ & $1.27(0.78,2.06)$ & $0.89(0.51,1.55)$ & $0.79(0.34,1.85)$ \\
\hline \multirow{2}{*}{$\begin{array}{l}\text { Symptoms only (C) } \\
\text { to sxHOA (D) }\end{array}$} & 38 (vs 27) & 18 (vs 47) & 41 (vs 24) & 7 (vs 11) & 10 (vs 24) & 7 (vs 20) \\
\hline & $1.46(0.91,2.36)$ & $1.49(0.86,2.59)$ & $1.07(0.65,1.78)$ & $1.41(0.63,3.12)$ & $2.38(1.23,4.63)$ & $2.25(1.08,4.70)$ \\
\hline \multicolumn{7}{|l|}{ Development of symptoms } \\
\hline \multirow{2}{*}{$\begin{array}{l}\text { No rHOA/symptoms (A) } \\
\text { to symptoms only (C) }\end{array}$} & 159 (vs 133) & 64 (vs 225) & 108 (vs 181) & 29 (vs 82) & 19 (vs 112) & 8 (vs 144) \\
\hline & $1.33(1.01,1.74)$ & $1.05(0.71,1.54)$ & $1.25(0.92,1.71)$ & $1.91(1.21,3.01)$ & $1.36(0.84,2.18)$ & $1.01(0.50,2.03)$ \\
\hline \multirow[t]{2}{*}{$\mathrm{rHOA}(\mathrm{B})$ to $\mathrm{s} \times \mathrm{HOA}(\mathrm{D})$} & 87 (vs 85) & 39 (vs 133) & 71 (vs101) & 17 (vs 48) & 15 (vs 71) & 6 (vs 81) \\
\hline & $0.93(0.63,1.35)$ & $1.29(0.78,2.15)$ & $1.08(0.71,1.63)$ & $0.95(0.52,1.73)$ & $0.83(0.43,1.61)$ & $0.98(0.38,2.51)$ \\
\hline \multicolumn{7}{|l|}{ Resolution of symptoms } \\
\hline \multirow{2}{*}{$\begin{array}{l}\text { Symptoms only (C) to } \\
\text { no rHOA/symptoms (A) }\end{array}$} & 164 (vs 137) & 67 (vs 234) & 107 (vs 194) & 34 (vs 85) & 26 (vs 115) & 14 (vs 151) \\
\hline & $0.88(0.67,1.14)$ & $0.74(0.51,1.08)$ & $0.61(0.45,0.82)$ & $0.56(0.36,0.86)$ & $0.65(0.41,1.04)$ & $0.46(0.25,0.84)$ \\
\hline \multirow[t]{2}{*}{ sxHOA (D) to rHOA (B) } & 104 (vs 70) & 38 (vs 136) & 72 (vs 102) & 23 (vs 41) & 10 (vs 58) & 8 (vs 80) \\
\hline & $0.85(0.58,1.23)$ & $0.64(0.38,1.08)$ & $0.74(0.50,1.09)$ & $0.62(0.35,1.10)$ & $0.35(0.17,0.71)$ & $0.44(0.21,0.94)$ \\
\hline
\end{tabular}

${ }^{1}$ Model includes effects for baseline values of birth year, study cohort, age, sex, race, and education and time-dependent values of obesity, DM, CVD, and hip injury

${ }^{2}$ Model includes effects for baseline values of birth year, study cohort, age, sex, race, and education and time-dependent values of hip injury, DM, group of obesity with no CVD, group of no obesity with CVD, and group of obesity with CVD

${ }^{3}$ Model includes effects for baseline values of birth year, study cohort, age, sex, race, and education and time-dependent values of hip injury, CVD, group of obesity with no DM, group of no obesity with DM, and group of obesity with DM

${ }^{4}$ Model includes effects for baseline values of birth year, study cohort, age, sex, race, and education and time-dependent values of hip injury, obesity, group of CVD with no DM, group of no CVD with DM, and group of CVD with DM

Italics indicate statistical significance (i.e., the $95 \% \mathrm{Cl}$ excludes 1 )

CVD and development of asymptomatic rHOA (states A to B; aHR for women $1.42,95 \%$ CI $[0.98,2.05$ ); for men aHR $1.08,95 \%$ CI $[0.53,2.19)]$. Among men only, there was a significant reduction in symptom resolution in the presence of rHOA for those with DM versus those without (states D to B, aHR $0.28,95 \%$ CI $[0.10,0.81]$ ), although the direction of the non-significant associations in women and for other outcomes was generally consistent. Resolution of symptoms (states $\mathrm{C}$ to $\mathrm{A}$, or $\mathrm{D}$ to $\mathrm{B}$ ) was less likely in the presence of CVD, but this association was only statistically significant in women.

When stratified by race (Table 4), the most striking difference was for African Americans with DM, who, compared to African Americans without DM, had nearly four times the hazard of developing incident symptomatic HOA (states C to D, aHR 3.57, 95\% CI [1.10, 11.7]) and had more than twice the hazard of developing symptoms when rHOA was present, although the latter was of borderline statistical significance (states B to D, aHR 2.09, 95\% CI $[0.97,4.54])$. Additionally, the significant association in the overall analysis between obesity and development of symptoms (states A to C) was seen only in white individuals; the association seen in women between CVD and incident rHOA was also evident only in whites (Table 4).

In sensitivity analyses of the results in Tables 2, 3, and 4 limited to those individuals with at least two follow-up time points, magnitudes of effects were not substantially changed (although some were no longer statistically significant due to smaller sample sizes).

\section{Discussion}

This longitudinal analysis using a state transition model identified several associations among common comorbidities and state transitions of HOA in a communitybased cohort. Obesity was associated with greater risk of developing symptoms, particularly in women, while CVD and DM reduced the hazard of symptom resolution over time. The effects were stronger for combinations of comorbidities, where most combinations of two comorbidities resulted in a statistically significantly lower hazard of symptom resolution. Additionally, in combination with DM, both obesity and CVD resulted in twice the hazard of incident sxHOA (compared to those without DM or either obesity or CVD). In stratified analyses, African Americans with DM, compared to 
Table 3 Adjusted hazard ratios (aHR) and 95\% confidence intervals (CI) for comorbid conditions, individually, on modeled transition states, over the full follow-up period, by sex

\begin{tabular}{|c|c|c|c|c|}
\hline \multirow[t]{3}{*}{ Strata } & \multirow[t]{3}{*}{ Type of transition } & Obesity (vs no obesity) $^{1}$ & \multirow[t]{3}{*}{ DM (vs no DM) ${ }^{1}$} & \multirow[t]{3}{*}{ CVD (vs no CVD) } \\
\hline & & \multirow{2}{*}{$\begin{array}{l}n \text { (vs } n) \text { for transitions } \\
\text { aHR }(95 \% \mathrm{Cl})\end{array}$} & & \\
\hline & & & & \\
\hline \multirow[t]{15}{*}{ Women } & \multicolumn{4}{|l|}{ Development of rHOA } \\
\hline & \multirow[t]{2}{*}{ No rHOA/symptoms (A) to rHOA (B) } & 56 (vs 77) & 25 (vs 108) & 49 (vs 84) \\
\hline & & $0.85(0.59,1.21)$ & $1.03(0.61,1.75)$ & $1.42(0.98,2.05)$ \\
\hline & \multirow[t]{2}{*}{ Symptoms only (C) to sxHOA (D) } & 28 (vs 21) & 13 (vs 36) & 32 (vs 17) \\
\hline & & $1.27(0.73,2.20)$ & $1.42(0.74,2.75)$ & $1.23(0.70,2.17)$ \\
\hline & \multicolumn{4}{|l|}{ Development of symptoms } \\
\hline & \multirow[t]{2}{*}{ No rHOA/symptoms (A) to symptoms only (C) } & 108 (vs 77) & 39 (vs 146) & 64 (vs 121) \\
\hline & & $1.44(1.02,2.02)$ & $0.92(0.56,1.50)$ & $1.22(0.83,1.79)$ \\
\hline & \multirow[t]{2}{*}{$r H O A(B)$ to $s \times H O A(D)$} & 55 (vs 59) & 26 (vs 88) & 51 (vs 63) \\
\hline & & $0.70(0.43,1.13)$ & $1.42(0.78,2.61)$ & $0.94(0.58,1.54)$ \\
\hline & \multicolumn{4}{|l|}{ Resolution of symptoms } \\
\hline & \multirow[t]{2}{*}{ Symptoms only (C) to no rHOA/symptoms (A) } & 122 (vs 86) & 48 (vs 160) & 75 (vs 133) \\
\hline & & $0.93(0.68,1.27)$ & $0.78(0.49,1.22)$ & $0.60(0.42,0.87)$ \\
\hline & \multirow[t]{2}{*}{ sxHOA (D) to rHOA (B) } & 69 (vs 49) & 26 (vs 92) & 52 (vs 66) \\
\hline & & $0.71(0.44,1.13)$ & $0.86(0.48,1.54)$ & $0.60(0.38,0.94)$ \\
\hline \multirow[t]{15}{*}{ Men } & \multicolumn{4}{|l|}{ Development of $\mathrm{rHOA}$} \\
\hline & \multirow[t]{2}{*}{ No rHOA/symptoms (A) to rHOA (B) } & 26 (vs 39) & 14 (vs 51) & 20 (vs 45) \\
\hline & & $0.93(0.55,1.57)$ & $1.10(0.52,2.31)$ & $1.08(0.53,2.19)$ \\
\hline & \multirow[t]{2}{*}{ Symptoms only (C) to sxHOA (D) } & 10 (vs 6) & 5 (vs 11) & 9 (vs 7) \\
\hline & & $2.14(0.88,5.21)$ & $1.26(0.46,3.47)$ & $0.68(0.21,2.17)$ \\
\hline & \multicolumn{4}{|l|}{ Development of symptoms } \\
\hline & \multirow[t]{2}{*}{ No rHOA/symptoms (A) to symptoms only (C) } & 48 (vs 56) & 25 (vs 79) & 44 (vs 60) \\
\hline & & $1.15(0.74,1.79)$ & $1.32(0.71,2.45)$ & $1.26(0.75,2.13)$ \\
\hline & \multirow[t]{2}{*}{$\mathrm{rHOA}(\mathrm{B})$ to $\mathrm{sxHOA}(\mathrm{D})$} & 32 (vs 26) & 13 (vs 45) & 20 (vs 38) \\
\hline & & $1.50(0.78,2.88)$ & $0.81(0.33,2.03)$ & $1.46(0.65,3.28)$ \\
\hline & \multicolumn{4}{|l|}{ Resolution of symptoms } \\
\hline & \multirow[t]{2}{*}{ Symptoms only (C) to no rHOA/symptoms (A) } & 42 (vs 51) & 19 (vs 74) & 32 (vs 61) \\
\hline & & $0.78(0.49,1.24)$ & $0.70(0.38,1.30)$ & $0.61(0.36,1.05)$ \\
\hline & \multirow[t]{2}{*}{ sxHOA (D) to rHOA (B) } & 35 (vs 21) & 12 (vs 44) & 20 (vs 36) \\
\hline & & $1.15(0.60,2.21)$ & $0.28(0.10,0.81)$ & $1.12(0.54,2.33)$ \\
\hline
\end{tabular}

${ }^{1}$ Model includes effects for baseline values of birth year, study cohort, age, race, and education and time-dependent values of obesity, DM, CVD, and hip injury Italics indicate statistical significance (i.e., the $95 \% \mathrm{Cl}$ excludes 1)

those without DM, had greater hazard for development of symptoms or sxHOA. These findings reinforce the effects of multiple chronic conditions in individuals with or at risk for HOA.

Higher BMI has been associated with greater selfreported pain and poorer function among individuals awaiting hip replacement surgery, despite similar radiographic severity of disease [12]. Some studies have reported no association between the metabolic syndrome or its individual components in severe HOA [6]. However, one or more comorbid conditions conferred a higher risk of revision of hip arthroplasty (HR 1.16, 95\% CI $[1.08,1.23])$ in a large Finnish registry; this was mostly attributable to CVD and particularly heart failure [4]. A recent systematic review of comorbidities and the prognosis of clinical symptoms in knee and/or hip OA noted greater pain and poorer performance-based function in those with one or more comorbid conditions; specifically, DM was associated with greater pain, while CVD was more associated with decrements in physical function [13]. This is consistent with our findings of persistent symptoms and greater chance of developing 
Table 4 Adjusted hazard ratios (aHR) and 95\% confidence intervals (CI) for comorbid conditions, individually, on modeled transition states, over the full follow-up period, by race

\begin{tabular}{|c|c|c|c|c|}
\hline \multirow[t]{2}{*}{ Strata } & \multirow[t]{2}{*}{ Type of transition } & Obesity (vs no obesity) ${ }^{1}$ & \multirow[t]{2}{*}{ DM (vs no DM) ${ }^{1}$} & \multirow[t]{2}{*}{ CVD (vs no CVD) } \\
\hline & & $\begin{array}{l}n(v s n) \text { for transitions } \\
\text { aHR }(95 \% \mathrm{Cl})\end{array}$ & & \\
\hline \multirow[t]{15}{*}{ White } & \multicolumn{4}{|l|}{ Development of rHOA } \\
\hline & \multirow[t]{2}{*}{ No rHOA/symptoms (A) to rHOA (B) } & 45 (vs 88) & 18 (vs 115) & 46 (vs 87) \\
\hline & & $0.75(0.51,1.11)$ & $0.93(0.48,1.80)$ & $1.49(0.99,2.24)$ \\
\hline & \multirow[t]{2}{*}{ Symptoms only (C) to sxHOA (D) } & 31 (vs 22) & 12 (vs 41) & 33 (vs 20) \\
\hline & & $1.48(0.89,2.44)$ & $1.16(0.61,2.23)$ & $1.02(0.58,1.77)$ \\
\hline & \multicolumn{4}{|l|}{ Development of symptoms } \\
\hline & \multirow[t]{2}{*}{ No rHOA/symptoms (A) to symptoms only (C) } & 107 (vs 107) & 46 (vs 168) & 76 (vs 138) \\
\hline & & $1.46(1.06,2.00)$ & $1.20(0.75,1.90)$ & $1.31(0.91,1.89)$ \\
\hline & \multirow[t]{2}{*}{$\mathrm{rHOA}(\mathrm{B})$ to $\mathrm{s} \times \mathrm{HOA}(\mathrm{D})$} & 65 (vs 72) & 25 (vs 112) & 57 (vs 80) \\
\hline & & $0.88(0.65,1.54)$ & $0.94(0.47,1.87)$ & $1.36(0.84,2.21)$ \\
\hline & \multicolumn{4}{|l|}{ Resolution of symptoms } \\
\hline & \multirow[t]{2}{*}{ Symptoms only (C) to no rHOA/symptoms (A) } & 100 (vs 99) & 38 (vs 161) & 69 (vs 130) \\
\hline & & $1.03(0.75,1.43)$ & $0.68(0.40,1.17)$ & $0.58(0.39,0.85)$ \\
\hline & \multirow[t]{2}{*}{ sxHOA (D) to rHOA (B) } & 73 (vs 59) & 29 (vs 103) & 55 (vs 77) \\
\hline & & $0.87(0.57,1.38)$ & $0.58(0.31,1.06)$ & $0.87(0.54,1.39)$ \\
\hline \multirow[t]{15}{*}{ Black } & \multicolumn{4}{|l|}{ Development of rHOA } \\
\hline & \multirow[t]{2}{*}{ No rHOA/symptoms (A) to rHOA (B) } & 37 (vs 28) & 21 (vs 44) & 23 (vs 42) \\
\hline & & $1.11(0.69,1.78)$ & $1.10(0.59,2.06)$ & $1.17(0.69,1.97)$ \\
\hline & \multirow[t]{2}{*}{ Symptoms only (C) to sxHOA (D) } & 7 (vs 5) & 6 (vs 6) & 8 (vs 4) \\
\hline & & $1.09(0.32,3.71)$ & $3.57(1.10,11.7)$ & $1.22(0.36,4.04)$ \\
\hline & \multicolumn{4}{|l|}{ Development of symptoms } \\
\hline & \multirow[t]{2}{*}{ No rHOA/symptoms (A) to symptoms only (C) } & 49 (vs 26) & 18 (vs 57) & 32 (vs 43) \\
\hline & & $1.00(0.59,1.72)$ & $0.79(0.40,1.57)$ & $1.19(0.66,2.17)$ \\
\hline & \multirow[t]{2}{*}{$\mathrm{rHOA}(\mathrm{B})$ to $\mathrm{s} \times \mathrm{HOA}(\mathrm{D})$} & 22 (vs 13) & 14 (vs 21) & 14 (vs 21) \\
\hline & & $0.69(0.32,1.46)$ & $2.09(0.97,4.54)$ & $0.63(0.27,1.49)$ \\
\hline & \multicolumn{4}{|l|}{ Resolution of symptoms } \\
\hline & \multirow[t]{2}{*}{ Symptoms only (C) to no rHOA/symptoms (A) } & 64 (vs 38) & 29 (vs 73) & 38 (vs 64) \\
\hline & & $0.66(0.42,1.04)$ & $0.77(0.46,1.31)$ & $0.70(0.43,1.13)$ \\
\hline & \multirow[t]{2}{*}{ sxHOA (D) to rHOA (B) } & 31 (vs 11) & 9 (vs 33) & 17 (vs 25) \\
\hline & & $0.69(0.35,1.39)$ & $0.91(0.37,2.24)$ & $0.52(0.25,1.09)$ \\
\hline
\end{tabular}

${ }^{1}$ Model includes effects for baseline values of birth year, study cohort, age, sex, and education and time-dependent values of obesity, DM, CVD, and hip injury Italics indicate statistical significance (i.e., the $95 \% \mathrm{Cl}$ excludes 1 )

symptomatic OA among those with multiple comorbidities including DM. Another study found a greater likelihood of persistent pain after joint replacement among individuals with $\mathrm{DM}$, but not with metabolic syndrome or obesity [14]. However, in the JoCo OA, the lifetime risk of HOA did not vary substantially by BMI or other demographic features [5].

In exploratory analyses stratified by sex, some of the associations were statistically significant only for women, but this may be due to limitations in sample size for the men, particularly since the effects were generally in the same direction. Interestingly, we did see a significant reduction in symptom resolution in men only for those with DM compared to those without. Because of the smaller numbers, we were not able to consider separately the four race by sex strata, and also we could not assess the effect of combinations of comorbidities in the sex and race strata.

In exploratory analyses stratified by race, we found an unexpected racial difference when modeling DM alone 
among African Americans: compared with those without $\mathrm{DM}$, individuals with DM were more likely to worsen from hip symptoms only to sxHOA, or from asymptomatic rHOA to sxHOA. This specific finding has not been previously reported. However, there is evidence that, compared with whites, African Americans with OA tend to report greater pain and have poorer self-reported function and greater disability; in some cases, these relationships are partly attenuated by differences in BMI, psychological factors (e.g., depression, pain coping, general health), or occupational exposures [15]. Similarly, compared with whites, African Americans have a greater risk, as well as overall poorer control, of DM and other cardiometabolic conditions (e.g., obesity, hypertension, metabolic syndrome) and are much more likely to develop end-stage renal disease (with or without DM); these disparities are hypothesized to occur partly because of differences in diet and physical activity, socioeconomic status, and access to care [16]. Therefore, this finding may relate to overall poorer management of these comorbid conditions among affected African Americans, who then are more likely to experience progression of $\mathrm{HOA}$, an area deserving of further study.

There are several limitations of this study. Our results in this community-based cohort of individuals in North Carolina may not be representative of other populations of differing ages or race/ethnicity. Additionally, the diagnoses of DM and CVD were based on participant selfreport of prior doctor diagnosis rather than direct testing (e.g., we do not have HbA1c values) or medical record review, although self-report of these conditions is fairly reliable $[17,18]$. Our data source is an important strength, as it is a racially diverse sample with 18 years of follow-up. Sensitivity analyses indicated no remarkable impact from loss to follow-up. We used an advanced and relatively novel statistical method, which has many benefits, including the ability to model several transitions of interest, rather than focusing on only one or a few outcomes, while still incorporating imprecise times of transition (i.e., interval-censored events, common in cohorts studying chronic disease). Here, we have simultaneously modeled incident rHOA, incident symptoms, incident sxHOA, and resolution of symptoms, allowing inclusion of a larger number of participants over multiple time points. This model also allows for inclusion of time-varying covariates, meaning that we were able to account for changes in obesity status, and new onset or resolution of comorbidities, over time.

\section{Conclusions}

Combinations of common comorbid conditions (i.e., obesity, DM, and CVD) led to higher likelihood of persistence and an increased chance of worsening symptoms or progression to sxHOA, most notably among African Americans with DM. The associations identified in this analysis highlight the combined impact of multiple comorbid conditions including $\mathrm{OA}$ and the need to consider multimorbidity in the evaluation and care of these patients.

\section{Abbreviations \\ aHR: Adjusted hazard ratio; BMI: Body mass index; Cl: Confidence interval; CVD: Cardiovascular disease; DM: Diabetes mellitus; HOA: Hip osteoarthritis: JoCo OA: Johnston County Osteoarthritis Project; KLG: Kellgren-Lawrence Grade; MSM: Markov multi-state models; OA: Osteoarthritis; rHOA: Radiographic hip osteoarthritis; SD: Standard deviation; sxHOA: Symptomatic hip osteoarthritis}

\section{Acknowledgements}

The authors would like to acknowledge the full research team and staff at UNC Thurston Arthritis Research Center and the participants and staff of the JoCo OA Project.

\section{Disclaimer}

The findings and conclusions in this report are those of the authors and do not necessarily represent the official position of the Centers for Disease Control and Prevention.

\section{Authors' contributions}

CA, AEN, YMG, JMJ, and LFC gave substantial contributions to the conception or design. CA, RJC, TAS, JBR, JMJ, and AEN gave substantial contributions to the acquisition, analysis, or interpretation of data. CA, AEN, LBM, LFC, and YMG gave substantial contributions to the initial draft preparation and revisions. All authors have read, commented on, and approved the submitted version. All authors agree to be accountable for their contributions and ensure the accuracy and integrity of the submitted work.

\section{Funding}

Funding for this project was provided in part by The Centers for Disease Control and Prevention (CDC: U01-DP003206 and DP006266, S043, S3486) and The National Institute of Arthritis and Musculoskeletal and Skin Diseases (NIAMS: P60-AR049465 and AR064166).

\section{Availability of data and materials}

The datasets used and/or analyzed during the current study are available from the corresponding author on reasonable request.

Ethics approval and consent to participate

The Johnston County Osteoarthritis Project has been approved by the University of North Carolina (IRB 92-0583).

Consent for publication

Not applicable.

\section{Competing interests}

The authors declare that they have no competing interests.

\section{Publisher's Note}

Springer Nature remains neutral with regard to jurisdictional claims in published maps and institutional affiliations.

\section{Author details}

${ }^{1}$ Thurston Arthritis Research Center, University of North Carolina at Chapel Hill, 3300 Doc J. Thurston Building, Campus Box \#7280, Chapel Hill, NC 27599-7280, USA. ${ }^{2}$ Department of Biostatistics, Gillings School of Global Public Health, University of North Carolina at Chapel Hill, $3106 \mathrm{E}$ McGavran-Greenberg Hall, Campus Box \#7420, Chapel Hill, NC 27599-7420, USA. ${ }^{3}$ Department of Radiology, University of North Carolina at Chapel Hill, 509 Old Infirmary Bldg, Campus Box \#7510, Chapel Hill, NC 27599-7510, USA. ${ }^{4}$ Centers for Disease Control and Prevention, 4770 Buford Highway NE MS S106-7, Atlanta, GA 30341, USA. ${ }^{5}$ School of Medicine, University of North 
Carolina at Chapel Hill, Chapel Hill, USA. ${ }^{6}$ Department of Epidemiology, Gillings School of Global Public Health, University of North Carolina at Chapel Hill, Chapel Hill, USA. ${ }^{7}$ Injury Prevention Research Center, University of North Carolina at Chapel Hill, Chapel Hill, USA. ${ }^{8}$ Division of Physical Therapy, Department of Allied Health, University of North Carolina at Chapel Hill, Chapel Hill, USA.

Received: 13 May 2019 Accepted: 9 January 2020

Published online: 20 January 2020

\section{References}

1. Hall AJ, Stubbs B, Mamas MA, Myint PK, Smith TO. Association between osteoarthritis and cardiovascular disease: systematic review and meta-analysis. Eur J Prev Cardiol. 2016;23(9):938-46.

2. Nuesch E, Dieppe P, Reichenbach S, Williams S, Iff S, Juni P. All cause and disease specific mortality in patients with knee or hip osteoarthritis: population based cohort study. BMJ. 2011;342:d1165.

3. Hawker GA, Croxford R, Bierman AS, Harvey PJ, Ravi B, Stanaitis I, Lipscombe LL. Allcause mortality and serious cardiovascular events in people with hip and knee osteoarthritis: a population based cohort study. PLoS One. 2014;9(3):e91286.

4. Jamsen E, Peltola M, Eskelinen A, Lehto MU. Comorbid diseases as predictors of survival of primary total hip and knee replacements: a nationwide register-based study of 96754 operations on patients with primary osteoarthritis. Ann Rheum Dis. 2013;72(12):1975-82

5. Murphy LB, Helmick CG, Schwart TA, Renner JB, Tudor G, Koch GG, Dragomir AD, Kalsbeek WD, Luta G, Jordan JM. One in four people may develop symptomatic hip osteoarthritis in his or her lifetime. Osteoarthr Cartil. 2010;18(11):1372-9.

6. Monira Hussain S, Wang Y, Cicuttini FM, Simpson JA, Giles GG, Graves S, Wluka AE. Incidence of total knee and hip replacement for osteoarthritis in relation to the metabolic syndrome and its components: a prospective cohort study. Semin Arthritis Rheum. 2014;43(4):429-36.

7. Veronese N, Trevisan C, De Rui M, Bolzetta F, Maggi S, Zambon S, Musacchio E, Sartori L, Perissinotto E, Crepaldi G, et al. Association of Osteoarthritis with Increased Risk of cardiovascular diseases in the elderly: findings from the Progetto Veneto Anziano study cohort. Arthritis Rheumatol. 2016;68(5):1136-44.

8. Liu B, Balkwill A, Banks E, Cooper C, Green J, Beral V. Relationship of height, weight and body mass index to the risk of hip and knee replacements in middle-aged women. Rheumatology (Oxford). 2007:46(5):861-7.

9. Jiang L, Rong J, Wang Y, Hu F, Bao C, Li X, Zhao Y. The relationship between body mass index and hip osteoarthritis: a systematic review and meta-analysis. Joint Bone Spine. 2011;78(2):150-5.

10. Jordan JM, Helmick CG, Renner JB, Luta G, Dragomir AD, Woodard J, Fang F, Schwartz TA, Abbate LM, Callahan LF, et al. Prevalence of knee symptoms and radiographic and symptomatic knee osteoarthritis in african americans and caucasians: the Johnston County Osteoarthritis Project. J Rheumatol. 2007;34:172-80.

11. Jackson $\mathrm{CH}$. Multi-state models for panel data: the msm package for R. J Stat Softw. 2011;38(8):1-28.

12. Lubbeke A, Duc S, Garavaglia G, Finckh A, Hoffmeyer P. BMl and severity of clinical and radiographic signs of hip osteoarthritis. Obesity (Silver Spring). 2009;17(7):1414-9.

13. Calders $P$, Van Ginckel A. Presence of comorbidities and prognosis of clinical symptoms in knee and/or hip osteoarthritis: a systematic review and meta-analysis. Semin Arthritis Rheum. 2018;47(6):805-13.

14. Rajamaki TJ, Jamsen E, Puolakka PA, Nevalainen PI, Moilanen T. Diabetes is associated with persistent pain after hip and knee replacement. Acta Orthop. 2015; 86(5):586-93.

15. Allen KD. Racial and ethnic disparities in osteoarthritis phenotypes. Curr Opin Rheumatol. 2010;22(5):528-32.

16. Ferdinand KC, Nasser SA. Racial/ethnic disparities in prevalence and care of patients with type 2 diabetes mellitus. Curr Med Res Opin. 2015;31(5):913-23.

17. Jackson JM, DeFor TA, Crain AL, Kerby TJ, Strayer LS, Lewis CE, Whitlock EP, Williams SB, Vitolins MZ, Rodabough RI, et al. Validity of diabetes self-reports in the Women's Health Initiative. Menopause. 2014;21(8):861-8.

18. Dey AK, Alyass A, Muir RT, Black SE, Swartz RH, Murray BJ, Boulos MI. Validity of selfreport of cardiovascular risk factors in a population at high risk for stroke. I Stroke Cerebrovasc Dis. 2015;24(12):2860-5.

\section{Ready to submit your research? Choose BMC and benefit from:}

- fast, convenient online submission

- thorough peer review by experienced researchers in your field

- rapid publication on acceptance

- support for research data, including large and complex data types

- gold Open Access which fosters wider collaboration and increased citations

- maximum visibility for your research: over $100 \mathrm{M}$ website views per year

At BMC, research is always in progress.

Learn more biomedcentral.com/submissions 